\title{
Propiedades Psicométricas de la Escala Breve de Bienestar Subjetivo en la Escuela para Adolescentes (BASWBSS) en una Muestra de Adolescentes Chilenos
}

\section{Psychometric Properties of the Brief Adolescents' Subjective Well-Being in School Scale (BASWBSS) in a Sample of Chilean Adolescents}

\author{
Mariavictoria Benavente D. ${ }^{1}$, Félix Cova ${ }^{2}$, Claudia P. Pérez- Salas ${ }^{2}$, Jorge J. Varela $^{3}$, Jaime Alfaro ${ }^{3}$ \\ y Josefina Chuecas ${ }^{3}$
}

\begin{abstract}
Resumen
En el presente artículo se exploran las propiedades psicométricas de la Escala Breve de Bienestar Subjetivo en la Escuela para Adolescentes en una muestra de 1332 estudiantes chilenos de 13 a 19 años. Se realizaron análisis de estructura factorial, consistencia interna y validez convergente. Los resultados muestran una estructura bifactorial, que coincide con la propuesta por los autores de la escala y con una consistencia interna y validez convergente adecuada. Los resultados indican que la BASWBSS es un instrumento de autoinforme adecuado para la medición de los niveles de Bienestar Subjetivo en la Escuela en adolescentes chilenos. Se discute sobre la importancia de contar con instrumentos cuyo uso en combinación con otras medidas relacionadas con la escuela permita tener una comprensión más profunda de los procesos ligados a la promoción de la salud mental en la adolescencia y para la evaluación y seguimiento de intervenciones preventivas en el contexto escolar.
\end{abstract}

Palabras clave: propiedades psicométricas, bienestar subjetivo en la escuela, adolescencia

\begin{abstract}
This article explores the psychometric properties of the Brief Adolescents' Subjective Well-Being in School Scale (BASWBSS) in a sample of 1332 Chilean students aged from 13 to 19 years. We performed factor structure, internal consistency and convergent validity analyses. The results show a bifactorial structure, which coincides with that proposed by the authors of this scale and has an internal consistency and adequate convergent validity. The results indicate that the BASWBSS is an adequate self-reporting instrument for the measurement of Chilean adolescents' Subjective Well-being levels in school. We discuss the importance of having instruments whose use, in combination with other measures related to school, allows for a deeper understanding of the processes related to the promotion of mental health in adolescence and for the assessment and follow-up of preventive interventions in the school context.
\end{abstract}

Keywords: psychometric properties, subjective wellbeing in school, adolescence

Agradecimientos: La presente investigación fue financiada con el Fondo Interno de Investigación de la Universidad del Desarrollo proyecto número 20151019132324046303

\footnotetext{
${ }^{1}$ Facultad de Psicología Universidad del Desarrollo, Concepción, Chile. Becaria CONICYT del Programa de Doctorado en Salud Mental, Universidad de Concepción, Ainavillo 456, Concepción, Chile, Tel.: 56412242549. Correo: mbenavented@udd.cl

${ }^{2}$ Departamento de Psicología Universidad de Concepción, Chile.

${ }^{3}$ Facultad de Psicología Universidad del Desarrollo, Santiago, Chile.
} 


\section{Introducción}

El bienestar subjetivo y, en particular, su componente cognitivo, la satisfacción con la vida, ha sido asociado a distintos resultados vinculados al desarrollo y la salud mental de los adolescentes. Así se evidencia que la alta satisfacción con la vida se relaciona positivamente con la salud física, la salud mental, las buenas relaciones interpersonales y el éxito educativo (Park, 2004). Además se relaciona con la ausencia de conductas de riesgo tales como el abuso de sustancias, la violencia, la agresión y la victimización sexual (Proctor, Linley, \& Maltby, 2009). Por el contrario, una menor satisfacción con la vida de los adolescentes se relaciona con diversos problemas psicológicos y sociales, tales como síntomas depresivos, comportamiento violento y agresivo, el uso de sustancias, intentos de suicidio, ideas de suicidio, baja autoestima y falta de armonía en las relaciones (Furr \& Funder, 1998; Suldo \& Huebner, 2004; Valois, Zullig, Huebner, \& Drane, 2009; Valois, Zullig, Huebner, \& Drane, 2004; Zullig, Valois, Huebner, Oeltmann, \& Drane, 2001). Se ha observado una posible función de la satisfacción con la vida como un amortiguador (buffer) contra los efectos negativos del estrés y desarrollo de conductas psicopatológicas (Suldo \& Huebner, 2004).

El bienestar subjetivo es un componente clave en el logro de una adecuada salud mental (Dominguez-Guedea, 2016) y es un factor determinante de muchos resultados positivos en la vida de niños, niñas y adolescentes. Así, la incorporación de medidas de satisfacción con la vida en la evaluación e implementación de programas educativos y sociales es una importante contribución para establecer los efectos diferenciales y el impacto de este tipo de servicios en la calidad de vida y la salud positiva de los jóvenes. (Proctor, Maltby, \& Linley, 2011).

$\mathrm{Si}$ bien el bienestar es un fenómeno individual, está siempre en conexión con el contexto interpersonal, social, familiar e institucional y presenta especificidades situacionales (McGraw, Moore, Fuller, \& Bates, 2008). De allí que la evaluación del bienestar subjetivo de niños y adolescentes debe atender a la diversidad de contextos vitales (escuela, familia, comunidad) en que se desenvuelven (Lee
\& Yoo, 2015; O’Hare, \& Gutierrez, 2012). La evaluación de estos dominios específicos de la vida de los adolescentes puede ser un aporte para entender el funcionamiento global actual de los adolescentes (Nickerson \& Nagle, 2004).

Para niños y adolescentes, la escuela representa un ámbito particularmente relevante en su vida, tanto por el tiempo diario que pasan en ella como por las oportunidades de aprendizaje y de experiencia que provee, constituyéndose en un ámbito relevante para la comprensión del bienestar y salud mental en la infancia y adolescencia (Liu, Mei, Tian, \& Huebner, 2015). Para Noddings (2003) una educación de buena calidad debe preocuparse no solo de los aprendizajes académicos sino también de la felicidad de sus estudiantes en la escuela y de su satisfacción escolar.

La satisfacción escolar es definida como la evaluación cognitiva y subjetiva sobre la calidad percibida de la vida escolar (Tian, 2008). Como un aspecto de la satisfacción con la vida en general, provee de una evaluación de un área importante en la vida diaria de los niños y adolescentes y ha probado ser un constructo relevante para la comprensión del funcionamiento de los adolescentes en la escuela y de su calidad de vida en general (Elmore \& Huebner, 2010; Eryilmaz, 2012; Huebner, 1994).

Se ha documentado que de los ámbitos de satisfacción con la vida de los estudiantes, la satisfacción con la escuela es el ámbito que recibe la menor valoración en comparación con los otros ámbitos de la vida de los adolecentes. Según Huebner, Drane, \& Valois (2000) un cuarto de los estudiantes encuestados en su estudio se sienten insatisfechos con la escuela. La insatisfacción con la escuela se ha argumentado como la razón más común para el abandono escolar, y, también, ha sido relacionada con la conducta en la escuela: a menor satisfacción con la escuela, mayor presencia de conductas internalizantes y externalizantes (Baker \& Maupin, 2009; De Santis King, Huebner, Suldo, \& Valois, 2007; Huebner, Gilman, Reschly, \& Hall, 2009).

Si bien a nivel global la relación entre la edad, nivel de escolaridad y satisfacción con la vida, es débil, se ha observado que la satisfacción con la escuela disminuye con la edad y curso. Niños y niñas en cursos avanzados de primaria $\mathrm{y}$ 
secundaria informan niveles positivos de satisfacción global con la vida; sin embargo, es en relación al ámbito escolar donde presentan mayor insatisfacción (Elmore \& Huebner, 2010; Huebner et al., 2000; Huebner, Valois, Paxton, \& Drane, 2005; Liu et al., 2015). Esta disminución se ha asociado al incremento de la carga académica y personal y la reducción de la atención personalizada de la secundaria (De Santis King et al., 2007).

La relación entre la satisfacción con la escuela y edad es sensible al género: la disminución de las puntuaciones en las escalas de satisfacción con la escuela comienza más temprano para las niñas que en los niños (Bradshaw, Keung, Rees, \& Goswami, 2011). Otros estudios han reportado que la satisfacción con la escuela, medida con la Escala Breve de Bienestar Subjetivo en la Escuela para Adolescentes (BASWBSS), es mayor en las mujeres que los hombres (Liu et al., 2015).

Por otra parte, una alta satisfacción con la escuela ha sido asociada con variables psicológicas positivas como la esperanza (Castro Solano, 2011; Huebner \& Gilman, 2006), locus de control (Huebner, Ash, \& Laughlin, 2001), afectos positivos (Verkuyten\&Thijs, 2002), satisfacción global con la vida (Huebner, 1994) y la autoestima (Huebner y Gilman, 2006). Y con bajos niveles de ansiedad, depresión y estrés (Huebner et al., 2001; Huebner \& McCullough, 2000; McGraw et al. 2008). También ha sido asociada significativamente a variables del ambiente; por ejemplo, los adolescentes que reciben apoyo de ambos padres están más satisfechos con la escuela (De Santis King et al., 2007; Elmore \& Huebner, 2010; Huebner et al., 2001; Nickerson \& Nagle, 2004; Whitley, Huebner, Hills, \& Valois, 2012). Sin embargo, la relación entre la satisfacción escolar y variables demográficas tales como género, etnia y nivel socioeconómico es débil (De Santis King et al., 2007; Elmore \& Huebner, 2010; Huebner et al. 2001).

Verkuyten y Thijs (2002) concluyen que las autopercepciones, como la autoestima social percibida y la competencia académica autopercibida, tienen un rol mediador en la relación entre la victimización en la escuela, resultados académicos y la satisfacción con la escuela. Sin embargo, señalan que la satisfacción escolar no solo está determinada por características individuales, porque los niños que comparten una clase y ciertas características de la estructura de la misma, tienen niveles similares de satisfacción escolar. Un 7.1\% de la variabilidad de los puntajes en satisfacción con la escuela es explicada por la clase a la que el niño pertenece. Asimismo los resultados muestran un efecto directo e independiente del clima académico en la satisfacción escolar. Los estudiantes que asisten a clases que tienen un buen clima académico y que les ofrecen oportunidades de aprender y desarrollar sus capacidades presentan mayor satisfacción con su escuela.

El profesor es una pieza clave en la satisfacción que los adolescentes sienten en su escuela. La satisfacción con la escuela correlaciona positivamente con todas las variables de apoyo social desagregado como apoyo de los padres, del profesor y de los compañeros de clase. Los estudiantes mostraron mejores niveles de satisfacción cuando los profesores muestran un rol activo en proteger el clima de su clase e intervienen tempranamente en situaciones de agresión escolar entre pares (Verkuyten \& Thijs, 2002).

Más recientemente Liu et al. 2015, encontró que el apoyo social percibido por el estudiante de parte de los profesores y compañeros de clase es fundamental para explicar la satisfacción con la escuela, destacándose como los predictores más relevantes de ésta. Sin embargo, la relevancia de una u otra varió según la edad. En los estudiantes de educación primaria (correspondiente a estudiantes de 9 a 12 años) y de enseñanza media superior (estudiantes de 16 a 18 años) fue más importante el apoyo del profesor, mientras que en el grupo educación media (edades de 13 a 15 años) fue más importante el apoyo de los compañeros de clase. Los estudiantes que experimentan más apoyo de sus compañeros de clase presentan una balanza de afectos más positiva que los que no lo experimentan de este modo.

Asimismo estos autores observaron que el género tiene un efecto moderador en la influencia del apoyo social en la satisfacción con la escuela. En el grupo de educación primaria, en los hombres tuvo más influencia el apoyo del profesor en la satisfacción con la escuela que en 
las mujeres. En el grupo de educación media: el apoyo de los compañeros de clase influía en la satisfacción con la escuela solo en mujeres y no así en hombres. En el grupo de los mayores no tuvo una influencia el género en la relación entre apoyo y satisfacción con la escuela (Liu et al., 2015).

La mayoría de los estudios sobre satisfacción con la escuela se han realizado con población de otros países tales como China (Liu et al. 2015; Tian, Liu, Huang, \& Huebner, 2013), Korea del Sur (Park, 2005), Estados Unidos (Okun, Braver, \& Weir, 1990) u Holanda (Verkuyten \& Thijs, 2002) y los autores coinciden en que los resultados muestran diferencias según países debido, en parte, a las características culturales que tienen los modelos educativos en las distintas naciones. En Chile, Alfaro et al. 2016 han informado que en estudiantes de 9 a 14 años el promedio de satisfacción con la escuela es de 7.95 en una escala de 0 a 10 puntos; no observaron diferencias significativas por sexo pero sí por curso: los estudiantes de quinto básico presentan mayor satisfacción con su escuela que los alumnos de cursos superiores. También observaron diferencias significativas por dependencia del establecimiento, los estudiantes de escuelas de dependencia municipal presentan mayor satisfacción que aquellos que asisten a escuelas de dependencia compartida o particulares.

El estudio de la satisfacción requiere el desarrollo de nuevas medidas, en particular medidas que permitan monitorear el bienestar en poblaciones objetivos específicas, además de contar con medidas que permitan hacer comparaciones transnacionales, y/o de grupos o entornos culturales diversos (Siyez \& Kaya, 2008; Casas, 2010; Casas, 2011). El presente estudio tiene como objetivo conocer las propiedades psicométricas de la Escala Breve de Bienestar Subjetivo en la Escuela en una muestra de adolescentes chilenos.

\section{Escala Breve de Bienestar Subjetivo en la Escuela para Adolescentes (BASWBSS)}

Basado en la propuesta de Diener (2006) sobre bienestar subjetivo compuesto por un componente cognitivo (satisfacción con la vida de manera global y por ámbitos) y un componente afectivo (balanza de afectos), Tian (2008) propone un modelo teórico sobre el bienestar subjetivo en la escuela, el cual representa cómo los estudiantes evalúan subjetiva y emocionalmente las experiencias que viven específicamente en el contexto escolar. En este modelo se incluye una evaluación cognitiva de la vida escolar en distintos ámbitos de esta (como por ejemplo, relación profesor- alumno, aprendizaje, etc.) y un componente afectivo, basado en la balanza de afectos positivos y negativos experimentados por el estudiante en la escuela. Bajo este modelo, la satisfacción escolar se refiere a la evaluación cognitiva que realiza el estudiante de distintos aspectos de su vida escolar y la balanza de afectos se refiere a los afectos positivos y negativos que experimenta el estudiante en su vida escolar. La satisfacción con la escuela es, en este sentido, un componente de un constructo mayor denominado bienestar subjetivo relacionado con la escuela (Tian, Chen, \& Huebner, 2013).

Basado en la ASWBSS (Adolescents' SubjectiveWell- Being in SchoolScale) propuesta por Tian (2008), Tian, Wang y Huebner (2015) desarrolla el BASWBSS (Brief Adolescents' Subjective Well-Being in School Scale) una versión abreviada de la escala inicial, que reduce los 50 ítems que contenía ASWBSS a 8 ítems. Los 6 primeros se presentan en una escala de acuerdo donde cada uno de los reactivos describe uno de los ámbitos de la satisfacción con la escuela (logro, administración escolar, relación profesorestudiante, relaciones con pares, metodologías de enseñanza y aprendizaje). Estos se responden en una escala tipo Likert de 6 puntos.

Para medir el componente afectivo del bienestar subjetivo en la escuela se incluyen dos reactivos, uno de ellos evalúa la presencia de afectividad positiva y el otro de afectividad negativa en el contexto escolar. Estos ítems se responden en una escala de frecuencia de 6 puntos. La Tabla 1 muestra los ítems de la escala original en inglés y sus dimensiones.

Esta escala ha sido utilizada en muestras desde 8 a 19 años, mostrando índices de confiabilidad que varían entre 0.74 y 0.85 para la escala de satisfacción con la escuela; para la escala de afectividad las correlaciones entre afectos positivos y negativos, son negativas y moderadas que varían desde -.34 a -.44 (Akin, Çetin, \& Özen, 2016; Tian et al., 2015; Tian, Zhang, Huebner, Zheng, \& Liu, 2015). Las 
Tabla 1. Ítems escala BASWBSS y dimensiones

\begin{tabular}{lc}
\hline SubscaleSchoolSatisfaction & Dimensión \\
\hline $\begin{array}{l}\text { I perform well in school } \\
\text { My school is provided with } \\
\text { good school rules and } \\
\text { facilities }\end{array}$ & Achievement \\
$\begin{array}{l}\text { I have good relationships } \\
\text { with my teachers }\end{array}$ & $\begin{array}{c}\text { Teacher- } \\
\text { studentrelationships } \\
\text { I get along well with } \\
\text { classmates }\end{array}$ \\
$\begin{array}{l}\text { The teachers' instructional } \\
\text { methods and quality are } \\
\text { good }\end{array}$ & Peer relationships \\
$\begin{array}{l}\text { The curriculum and } \\
\text { homework assigned are } \\
\text { reasonable }\end{array}$ & Teaching \\
\hline SubscaleAffect in School & Academiclearning \\
\hline $\begin{array}{l}\text { In school, the frequency of } \\
\text { my pleasant feelings is } \\
\text { In school, the frequency of } \\
\text { my unpleasant feelings is }\end{array}$ & Dimensión \\
\hline
\end{tabular}

correlaciones ítem- total varían entre .25 y .62 (Akin et al. 2016).

La estructura que ha mostrado la escala es de dos factores, los cuales explican un total un $54.7 \%$ de la variabilidad de los puntajes (Tian, Wang et al., 2015; Tian, Zhang, et al., 2015). En mediciones test- retest con un periodo de 5 semanas ha mostrado correlaciones altas y significativas que varían entre .43 y .71 . Respecto de la validez convergente con la versión extendida de la escala, muestra correlaciones que varían entre .65 y .74 entre las dimensiones de la subescala de satisfacción con la escuela y de .72 para los afectos positivos y .67 para los afectos negativos de la subescala afectos en la escuela (Tian, Wang et al., 2015).

\section{Método}

\section{Diseño}

El presente estudio de tipo transversal y correlacional y debido a que el propósito es presentar las propiedades psicométricas de una escala, el diseño de investigación empleadoes de tipo instrumental (Ato, López, \& Benavente, 2013).

\section{Participantes}

En este estudio participaron 1.332 estudiantes, 760 mujeres $(57.1 \%)$ y 572 hombres $(42.9 \%)$ de entre 13 y 19 años $(\mathrm{M}=15.3$ años, $\mathrm{SD}=1.06)$. Todos estudiantes de primer, segundo o tercer año de Enseñanza Media (66 cursos en total) de 22 establecimientos educacionales privados, particulares subvencionados o municipales de la ciudad de Concepción, Chile. La Tabla 2 contiene información sociodemográfica de la muestra.

Tabla 2. Descripción sociodemográfica de los

\begin{tabular}{lll}
\multicolumn{3}{c}{ participantes } \\
\hline Variable & $\mathrm{n}$ & $\%$ \\
\hline Sexo & & \\
$\quad$ Hombre & 572 & 42.9 \\
$\quad$ Mujer & 760 & 57.1 \\
Edad & & \\
13 & 19 & 1.4 \\
14 & 311 & 23.3 \\
15 & 396 & 29.7 \\
16 & 459 & 34.5 \\
17 & 113 & 8.5 \\
18 & 27 & 2 \\
19 & 7 & 0.5 \\
Nivel & & \\
Primero medio & 484 & 36.3 \\
Segundo medio & 430 & 32.3 \\
$\quad$ Tercero medio & 418 & 31.4 \\
Dependencia & & \\
$\quad$ Particular & 289 & 21.7 \\
Particular subvencionado & 376 & 28.2 \\
$\quad$ Municipal & 667 & 50.1 \\
\hline
\end{tabular}

\section{Instrumentos}

\section{Escala Breve de Bienestar Subjetivo en la} Escuela para Adolescentes (BASWBSS) (Tian, Wang et al., 2015). La escala cuenta con dos subescalas, una de satisfacción escolar (School Satisfaction), que consta de 6 ítems, que explora el nivel de acuerdo del estudiante con su satisfacción en relación a cada una de las dimensiones de la vida escolar que el instrumento explora. La segunda subescala, de afectos en la escuela (Affect in School), presenta dos ítems que tienen como objetivo medir el componente afectivo del Bienestar Subjetivo en la escuela, a través del balance entre los afectos positivos y negativos predominantes en el contexto escolar. En la Tabla 3 se muestran los ítems en español y las dimensiones que exploran. El puntaje final de la subescala de satisfacción con la escuela se obtiene promediando los puntajes de los 6 ítems y el puntaje de la subescala de afectos en la escuela se obtiene restando el puntaje de los afectos negativos a los afectos positivos. Finalmente los puntajes de la subescala de satisfacción con la escuela y de la subescala de afectos en la escuela 
se suman obteniendo el puntaje del Bienestar Subjetivo en la escuela.

\section{Escala de Satisfacción vital de los estudiantes} (SLSS) Instrumento creado por Huebner (1991) para medir la satisfacción global con la vida en niños y adolescentes de 8 a 18 años. Consta de 7 ítems con 6 opciones de respuesta tipo Likert. La suma de puntajes de cada respuesta entrega un índice global de satisfacción que puede ir entre 7 a 42 puntos. Diversos estudios (Huebner, 1991; Galindez \& Casas, 2010; Weber, Ruch, \& Huebner, 2013) han reportado altos índices de confiabilidad $(\alpha=.79-.89)$. En una investigación comparativa entre países en la que participó Chile, se reportó un $\alpha$ de .77 (Casas, et al., 2015). Esta escala fue utilizada para la evaluación de la validez convergente de la BASWBSS.

\section{Procedimiento}

Los ítems fueron traducidos al español utilizando el método de traducción cruzada. Posteriormente, estos fueron sometidos a entrevistas cognitivas (Simth- Castro \& Molina, 2011) a un grupo de estudiantes que contaban con las características de la muestra definitiva. Considerando el criterio de saturación, se realizaron un total de 10 entrevistas. Este proceso indicó que el ítem 2 debía ser modificado de Myschoolisprovidedwithgoodschool rules and facilities, este fue traducido y adaptado como Mi colegio/liceo tiene un buen reglamento y $\mathrm{Mi}$ colegio/liceo tiene una buena infraestructura. Debido a que en el sistema de educación chileno cada uno de estos conceptos (reglamento e infraestructura) no dependen de la misma administración, resultaba confuso para los estudiantes establecer un nivel de acuerdo con la administración cuando las reglas dependen del establecimiento y la infraestructura puede depender del municipio o de la administración de fondos realizada por particulares con distintos grados de participación de la comunidad educativa (Ministerio de Educación, 2004), así la subescala Satisfacción con la Escuela quedó finalmente con 7 ítems.

Tanto las instrucciones como las categorías de respuestas se mantuvieron igual al instrumento original.
Para la selección de participantes, se tomó contacto con los establecimientos de la comuna de Concepción, una vez obtenida la autorización de los establecimientos, fueron seleccionados al azar un curso para cada nivel (primero, segundo y tercero medio), se contactó a los padres o apoderados de los estudiantes y se solicitó su consentimiento informado. A los estudiantes les fue solicitado el asentimiento informado. Para todos efectos se siguieron las indicaciones éticas de la Comisión Nacional de Investigación en Ciencia y Tecnología (Conicyt, 2007).

La aplicación de los instrumentos se realizó en la jornada regular de clases con la presencia del profesor del curso y de un miembro del equipo investigador con el objetivo de resguardar la calidad de los datos obtenidos y responder las inquietudes que pudieran surgir en los participantes. La modalidad utilizada fue de tipo autoadministrada. El tiempo de aplicación fue de aproximadamente 45 minutos. Los datos fueron recogidos durante el primer semestre del año 2016.

\section{Plan de Análisis}

El análisis de datos se realizó utilizando el software estadístico para las ciencias sociales (SPSS) versión 20. Para el análisis factorial confirmatorio (AFC) se utilizó el estimador ML (máxima verosimilitud) y programa Mplus 7.11 (Muthen \& Muthen, 2013).

Los datos fueron ingresados a través del proceso de doble digitación, se inspeccionaron los datos faltantes, para estos se realizó el proceso de imputación por la media.

El contraste de hipótesis se realizó en base a un nivel de confianza de un $95 \%$.

\section{Resultados}

\section{Descripción de las respuestas}

Se examinaron en primer término las características de cada ítem, así como el puntaje total de la BASWBSS con el fin de evaluar el ajuste a una distribución normal. En la Tabla 3 se puede observar una asimetría que fluctúa entre 1.56 a .33. Los valores de la curtosis oscilaron entre -.80 y 2.17. En este estudio se ha considerado el criterio propuesto por Kline (2011) para dar cumplimiento al supuesto de normalidad; 
Tabla 3. Promedios, desviación estándar, asimetría y curtosis, de los ítems y puntaje total la BASWBSS

\begin{tabular}{|c|c|c|c|c|}
\hline Ítems & $\mathrm{M}$ & $\mathrm{DE}$ & Asimetría & Curtosis \\
\hline Me va bien en mi colegio/liceo & 4.34 & 1.13 & -0.46 & .06 \\
\hline Mi colegio/liceo tiene un buen reglamento & 4.34 & 1.40 & -0.68 & .30 \\
\hline Mi colegio/liceo tiene una buena infraestructura & 4.38 & 1.48 & -0.75 & -.34 \\
\hline Tengo buenas relaciones con mis profesores/as & 4.81 & 1.26 & -1.10 & .72 \\
\hline Me llevo bien con mis compañeros/as & 5.09 & 1.18 & -1.56 & 2.17 \\
\hline $\begin{array}{l}\text { La calidad de enseñanza de los profesores/as es } \\
\text { buena }\end{array}$ & 4.48 & 1.41 & -.89 & .05 \\
\hline $\begin{array}{l}\text { La exigencia de las tareas que nos dan es } \\
\text { adecuada }\end{array}$ & 4.39 & 90 & -.50 & .02 \\
\hline Me siento bien en mi colegio/liceo & 4.46 & 1.27 & -.06 & -.80 \\
\hline Me siento mal en mi colegio/liceo & 2.30 & 1.20 & .33 & -.42 \\
\hline Puntaje total BASWBSS & 6.82 & 2.67 & -.33 & -.15 \\
\hline
\end{tabular}

Tabla 4. Cargas factoriales para los factores de la BASWBSS

\begin{tabular}{lcc}
\hline Item/ Escala & $\begin{array}{c}\text { Satisfacción con la } \\
\text { escuela }\end{array}$ & Afectos en la escuela \\
\hline I1 & $.40^{* *}$ & \\
I2 & $.67^{* *}$ & \\
I3 & $.55^{* *}$ & \\
I4 & $.71^{* *}$ & \\
I5 & $.55^{* *}$ & \\
I6 & $.77^{* *}$ & \\
I7 & $.56^{* *}$ & $.79^{* *}$ \\
I8 & & $-.020^{*}$ \\
I9 & & \\
\hline
\end{tabular}

Nota. $\mathrm{N}=1332 . *<.05, * *<.001$

${ }^{\text {a }}$ Solución estandarizada

en base a este criterio, las variables se ajustan a una distribución normal.

En la Tabla 3 se encuentran también los promedios y desviación estándar para los 7 ítems de la subescala satisfacción con la escuela, para los dos ítems de la subescala afectos en la escuela y para el puntaje total de bienestar subjetivo en la escuela. Como muestran los resultados el ítem que recibe mayor nivel de satisfacción es Me llevo bien con mis compañeros/as y los que reciben menor grado de desacuerdo son Me va bien en mi colegio/liceo y $\mathrm{Mi}$ colegio/liceo tiene un buen reglamento. En términos globales, un $22.5 \%$ se encuentra insatisfecho con la escuela y un $22 \%$ de los estudiantes presenta una balanza de afectos negativa o cero.

\section{Propiedades Psicométricas de la Escala}

\section{Análisis Factorial}

El análisis factorial confirmatorio mostró un ajuste adecuado a los datos $\left(\chi^{2}=201.668, \mathrm{gl}=27\right.$,

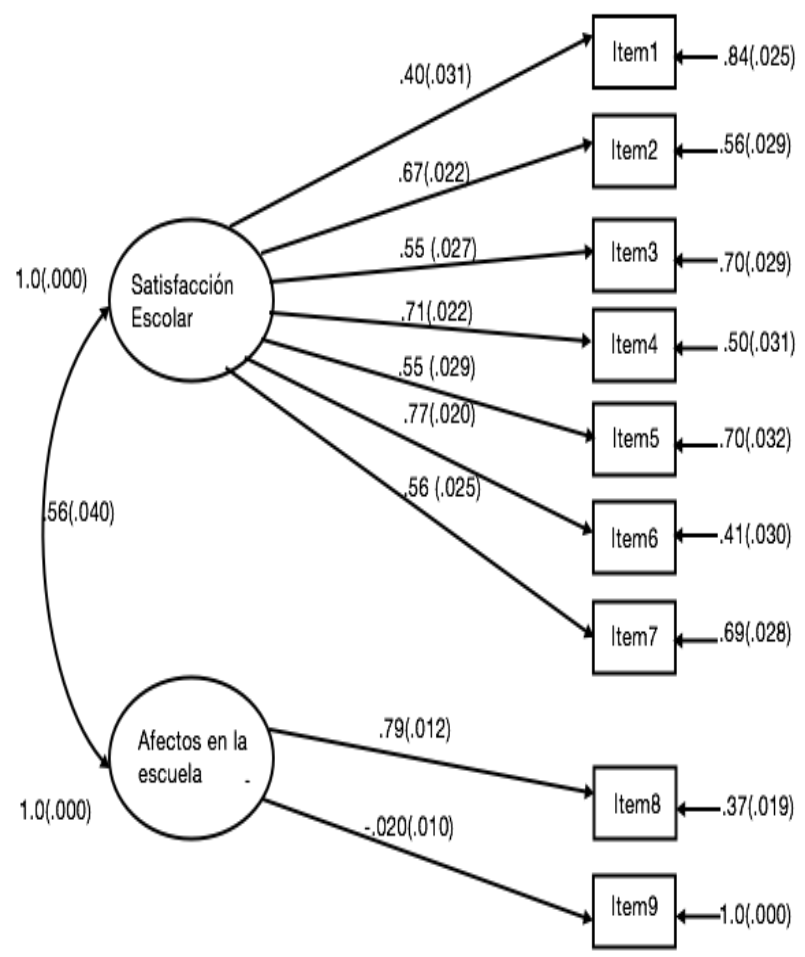

Figura 1. Diagrama con la estructura factorial del instrumento 
$p<.01$; CFI= .923; RMSEA $=.070$ [90\% C.I. .061.079]; SRMR=.040). El valor RMSEA es levemente superior a los estándares recomendados (i.e., RMSEA $=.06 \quad(\mathrm{Hu} \&$ Bentler, 1999); RMSEA=.07 (Steiger, 2007). Las cargas de los ítems a los factores son todas significativas y sobre .399, excepto la del ítem 9 al Factor 2, la cual es -.02 (M=.556) (ver Tabla 4). La Figura 1 muestra el diagrama con la estructura del instrumento.

\section{Indicadores de fiabilidad del instrumento}

En la Tabla 5 se presentan las correlaciones para la subescala satisfacción con la escuela entre los ítems de la prueba y de estos con el puntaje total de la subescala y de la prueba. Los ítems presentan correlaciones positivas aceptables entre ellos que van desde .17 a .57 . Las correlaciones entre los ítems y los puntajes totales de la subescala satisfacción con la escuela presentaron coeficientes positivos y elevados variando desde .50 a .77 . Se analizaron las correlaciones entre cada ítem y el puntaje total de la prueba con el objetivo de identificar ítems que no estuvieran asociados con la escala. Todos los ítems presentan adecuadas y positivas correlaciones con el puntaje total, éstas varían entre .39 y.52.

Tabla 5. Correlaciones entre los ítems y el puntaje total para la subescala satisfacción escolar

\begin{tabular}{lccccccc}
\hline Ítem & 1 & 2 & 3 & 4 & 5 & 6 & 7 \\
\hline 1 & - & & & & & & \\
2 & .23 & - & & & & & \\
3 & .17 & .50 & - & & & & \\
4 & .36 & .44 & .35 & - & & & \\
5 & .26 & .31 & .22 & .41 & - & & \\
6 & .28 & .51 & .43 & .57 & .43 & - & \\
7 & .17 & .40 & .30 & .34 & .32 & .45 & - \\
P S E & .50 & .74 & .66 & .74 & .61 & .77 & .66 \\
PT & .39 & .50 & .38 & .51 & .52 & .49 & .40 \\
\hline
\end{tabular}

Nota. PSE $=$ Puntaje subescala satisfacción con la escuela, $\mathrm{PT}=$ Puntaje total Bienestar Subjetivo en la Escuela. Todas las correlaciones son significativas al .01

En la Tabla 6 se presentan las correlaciones entre ítems de la subescala afectos en la escuela con el puntaje total de la subescala y con el puntaje total de la prueba. La correlación entre los dos ítems es negativa y alta, coincidiendo con el sentido en el que está construido por los autores (afectos positivos $\mathrm{y}$ afectos negativos). Las correlaciones de ambos ítems tanto con la subescala como con el puntaje total son elevadas.

Tabla 6. Correlaciones entre los ítems y el puntaje total para la subescala afectos en la escuela

\begin{tabular}{lcc}
\hline Ítem & 8 & 9 \\
\hline 8 & - & \\
9 & -.58 & - \\
P A E & .90 & -.88 \\
PT & .89 & -.83 \\
\hline
\end{tabular}

Nota. PAE= Puntaje subescala Afectos en la Escuela, $\mathrm{PT}=$ Puntaje total Bienestar Subjetivo en la Escuela. Todas las correlaciones son significativas al .01

El índice de confiabilidad alfa de Cronbach de la subescala satisfacción con la escuela fue de .79 mostrando una alta consistencia interna para la muestra en la que la escala fue puesta a prueba.

\section{Validez Convergente}

Para evaluar la validez convergente, se realizó una correlación bivariada de Pearson entre los puntajes totales de la BASWBSS y los de la Escala de Satisfacción con la vida de los Estudiantes (SLSS) obteniéndose una asociación positiva y moderada entre estas medidas ( $r(1283)$ $=.46, p<.01)$ lo cual apoya la validez convergente de la BASWBSS.

\section{Conclusión}

A partir de los resultados expuestos en el presente artículo, es posible señalar que la Escala Breve de Bienestar Subjetivo en la Escuela, presenta propiedades psicométricas adecuadas para ser utilizada en el contexto chileno. Esta escala, breve, permite acceder a la evaluación subjetiva de distintos aspectos de la vida escolar: logro académico, administración escolar, relación profesor- alumno(a), relación con pares y metodologías del aprendizaje usadas por los docentes. Además cuenta con una balanza de afectos, todo lo cual permite evaluar el componente cognitivo y afectivo del bienestar subjetivo.

Considerando que el contexto escolar es de alta relevancia para el desarrollo de los estudiantes y que el bienestar subjetivo ha sido ampliamente asociado a variables de salud mental positiva y ajuste social, contar con un instrumento 
breve y sencillo que permita su evaluación, nos parece un aporte para el seguimiento y el establecimiento de nuevas asociaciones entre variables escolares y el bienestar en la adolescencia. Así como también en la puesta a prueba de intervenciones destinadas a promover el bienestar desde el contexto escolar, este instrumento podría ser utilizado para evaluar los resultados de estas intervenciones, contando con una medida que permitiría realizar seguimiento a estas iniciativas.

\section{Discusión}

El presente artículo tuvo como objetivo evaluar las propiedades psicométricas de la Escala Breve de Bienestar Subjetivo en la Escuela (BASWBSS) elaborada por Tian, Wang et al. (2015), en una muestra de 1332 estudiantes chilenos de entre 13 y 19 años. Los resultados encontrados muestran que la escala presenta propiedades psicométricas adecuadas para ser utilizada en población adolescente chilena.

Sobre la estructura interna del instrumento los resultados muestran una composición bifactorial, que coincide con el propuesta que hacen los autores. Un factor corresponde al componente cognitivo, satisfacción con la escuela y otro refiere a un componente afectivo. En su conjunto estas dimensiones explican un $42.8 \%$ de la variabilidad de los puntajes de la escala. Este valor es un poco menor a lo reportado previamente por los autores de la escala que indican que la solución de dos factores explica un $54.7 \%$ de la varianza (Tian, Wang et al. 2015). El nivel de confiabilidad, medido a través del alfa de Cronbach fue .79. Esta se encuentra dentro del rango de los niveles de confiabilidad reportados en otros estudios. Las correlaciones entre los ítems y el puntaje total varió desde .39 y .52 que está en el rango de las correlaciones encontradas en estudios previos que la sitúan entre .25 y .62 (Akin et al. 2016; Tian, Wang et al., 2015; Tian, Zhang et al.2015).

Conocer estas dos dimensiones del bienestar subjetivo en la escuela es útil porque permite conocer dos aspectos de la experiencia de los estudiantes con la escuela. La satisfacción con la escuela informa sobre aspectos cognitivos del bienestar subjetivo, que pueden ser predictores de fenómenos como la deserción escolar (Huebner et al. 2000). Componentes más afectivos son también importantes porque se asocian con variables de clima social escolar en la medida que reportan cómo se sienten los alumnos/as dentro del colegio. El clima escolar se puede comprender como la percepción que un individuo tiene a partir de su experiencia subjetiva en un sistema escolar determinado. Este constructo entrega información significativa sobre la experiencia del estudiante en la escuela, ya que permite conocer su percepción acerca de las relaciones sociales en la comunidad escolar, así como la motivación, la implicación y el compromiso de los alumnos con este entorno (Aron, Milicic, \& Armijo, 2010; Martínez-Ferrer, Povedano-Díaz, Amador-Muñoz, \& Moreno- Ruiz, 2012).

Los estudios de satisfacción con la escuela y el bienestar en general son cada vez más recientes en América Latina (Alfaro et al., 2016), lo cual ha servido para conocer más sobre este fenómeno en la región. A su vez, permite identificar poblaciones en riesgo de acuerdo a los datos recolectados. De esta forma, llama la atención que un $22.5 \%$ de los estudiantes se encuentra insatisfecho con su escuela y un $22 \%$ tiene una balanza de afectos negativa o cero. Este resultado es concordante con los hallazgos de Huebner et al. (2000) que señalan que un cuarto de los estudiantes se encuentra insatisfecho con su escuela. Este resultado informa sobre la importancia de desarrollar programas de promoción y prevención en temas de salud mental en la escuela, lo cual implica adaptar las metodologías en temas de prevención y promoción de salud (Proctor et al. 2009), y resalta la necesidad del uso de instrumento debidamente validados en el contexto chileno.

Sobre los niveles de bienestar subjetivo en la escuela reportados por la muestra, la media para la subescala de satisfacción con la escuela fue 4,62 $(\mathrm{SD}=0.86)$ puntos, mientras que para la subescala afectos en la escuela fue de $2.16(\mathrm{SD}=2.18)$ y la media del bienestar subjetivo en la escuela fue de $6.77(\mathrm{SD}=2.66)$ en una escala que puede ir entre 5 y 12 puntos, en la presente muestra el puntaje mínimo fue -4 puntos y el máximo fue 11 puntos. en comparación con los resultados reportados por Tian, Wang et al. (2015) en el sudeste de China, la media de los puntajes de satisfacción con la 
escuela, afectos en la escuela y bienestar subjetivo los resultados fueron levemente superiores para los estudiantes chilenos.

El ítem que recibe mayor grado de acuerdo y satisfacción es Me llevo bien con mis compañeros coincidiendo con lo reportado por Liu et al., 2015 que indica que entre los 13 y 15 años el ámbito que recibe mayor importancia es la satisfacción con los compañeros de clase, mientras que los ítems que reciben menor satisfacción son Me va bien en mi colegio/liceo y Mi colegio/ liceo tiene un buen reglamento. Este resultado puede ser explicado por la edad de los sujetos de la muestra, en donde los pares juegan un rol fundamental y es concordante con lo planteado por otros autores en términos de que la alta satisfacción con los compañeros de clase (Casas, Baltatescu, Bertran, Gonzalez, \& Hatos, 2013) y la calidad de la relación con los pares (Leung \& Zhang, 2000) está relacionada con la satisfacción con la vida escolar, en ese sentido la incorporación de metodologías de tipo colaborativas puede potenciar el rol de la relación con los pares como un factor promotor de la salud mental.

Como limitaciones del estudio, es posible señalar que solo consideró población adolescente urbana escolarizada, de la ciudad de Concepción, dejando fuera criterios importantes como etnia, ruralidad, diversidad sexual, entre otras variables importantes para la comprensión del bienestar subjetivo en la escuela. En este sentido, los resultados no pueden ser generalizados para poblaciones diferentes a las consideradas en la muestra de este estudio. Otra limitación fue el diseño transversal del estudio, el cual no permitió realizar análisis longitudinales. Por ende, futuros usos de la escala podría considerar el levantamiento de datos a lo largo del tiempo para comparar la variación de la escala. Creemos que estos hallazgos pueden ser valiosos porque aportan evidencia de validez y fiabilidad de un instrumento breve, que explora un ámbito en la vida de los adolescentes de alta relevancia para la comprensión del bienestar subjetivo.

\section{Referencias}

Alfaro, J., Guzmán, J., Sirlopú, D., Reyes, F., Varela, J., \& Oyarzún, D. (2016). Bienestar subjetivo de la infancia en Chile en el contexto internacional. Santiago: Ediciones Universidad del Desarrollo. Recuperado de: http://psicologia.udd.cl/files/2016/12/bienestar -subjetivo-de-la- infancia.pdf

Akin, A., Çetin, R., \& Özen, Y. (2016). The validity and reliability of Turkish Version of the Brief Adolescents' Subjective Well- Being in School Scale. Journal of European Education, doi:10.18656/jee.15093

Ato, M., López, J. \& Benavente, A. (2013). Un sistema de clasificación de los diseños de investigación en psicología. Anales de Psicología, doi:10.6018/analesps.29.3.178511

Aron, A. M., Milicic, N., \& Armijo, I. (2012). Clima social escolar: Una escala de evaluación-Escala de Clima Social Escolar, ECLIS. Universitas Psychologica, 11(3), 803$813 . \quad$ Recuperado de: http://www.javeriana.edu.co/universitaspsych ologica/articulo.php?art=749

Bradshaw, J., Keung, A., Rees, G., \& Goswami, H. (2011). Children's subjective well being: International comparative perspectives. Children and Youth Services Review, doi:10.1016/j.childyouth.2010.05.010.

Baker, J. A., \& Maupin, A. N. (2009). School satisfaction and children's positive school adjustment. In R. Gilman, E. S. Huebner, \& M. J. Furlong (Eds.), Handbook of positive psychology in the schools (pp. 189196). New York: Routledge.

Casas, F. (2010). El bienestar personal: Su investigación en la infancia y la adolescencia. Encuentros en Psicología Social 5(1), 85-101.

Casas, F. (2011). Subjective social indicators and child and adolescent well-being. Child Indicators Research. doi:10.1007/s12187-010-9093-z

Casas, F., Alfaro, J., Sarriera, J., Bedin L., Grigoras, B., Baltatescu, S., Malo, S. \& Sirlopú, D. (2015). El bienestar subjetivo en la infancia: Estudio de la comparabilidad de 3 escalas psicométricas en 4 países de habla latina. Psicoperspectivas. doi:10.5027/PSICOPERSPECTIVASVOL14-ISSUE1- FULLTEXT-522

Casas, F., Baltatescu, S., Bertrán, I., González, M. \& Hatos, A. (2013). School satisfaction among adolescents: Testing different indicators for its measurement and its relationship with overall life satisfaction and subjective well-being in Romania and Spain. Social Indicators Research, doi:10.1007/s11205-012-0025-9

Castro Solano, A. (2011). Las rutas de acceso al bienestar. Relaciones entre bienestar hedónico y eudaimónico. Un estudio en población argentina. Revista Iberoamericana de 
Diagnóstico y Evaluación - e Avaliação Psicológica, 31(1), 37-57. Recuperado de http://www.redalyc.org/articulo.oa?id=459645 439003

Comisión Nacional de Investigación Científica y Tecnológica. (2007). Marcos Normativos en Ética de la Investigación Científica con seres vivos. Recuperado de http://www.conicyt.cl/fondecyt/files/2012/10/ Libro-2-Marcos-normativos-en-\%C3\%A9ticade-la-investigaci\%C3\%B3ncient\%C3\%ADfica-con-seres-vivos.pdf

De Santis King, A. L., Huebner, S., Suldo, S. M., $\&$ Valois, R. F. (2007). An ecological view of school satisfaction in adolescence: Linkages between social support and behavior problems. Applied Researchin Quality of Life. doi:10.1007/s11482-007-9021-7.

Diener, E. (2006). Guidelines for national indicators of subjective well-being and illbeing. Applied Research in Quality of Life, doi:10.1007/s10902-006-9000-y

Dominguez-Guedea, M. (2016). Bienestar en cuidadores familiares de adultos mayores: Un derecho, una aspiración y un constructo. Revista Iberoamericana de Diagnóstico y Evaluación Psicológica - e Avaliação Psicológica, 41(1), 104-117.Recuperado de: http://www.aidep.org/03_ridep/R41/Art9.pdf

Elmore, G. \& Huebner, E. S. (2010). Adolescents' satisfaction with school experiences: Relationships with demographisc, attachment relationships, and school engagement behavior. Psychology in the Schools. doi:10.1002/pits.20488.

Eryilmaz, A. (2012). A model for subjective wellbeing in adolescence: Need satisfaction and reasons for living. Social Indicators Research, doi:10.1007/s11205-011-9863-0

Furr, R.M. \& Funder. D (1998). A multimodal analysis of personal negativity. Journal of Personality and Social Psychology. doi:10.1037/0022- 3514.74.6.1580

Galindez, E. \& Casas, F. (2010). Adaptación y validación de la Students' Life Satisfaction Scale (SLSS) con adolescentes. Estudios de Psicología, 31(1), 79-87. Recuperado de: http://www.tandfonline.com/toc/redp20/31/1? nav $=$ tocList

Hu, L. T., \& Bentler, P. M. (1999). Cutoff criteria for fit indexes in covariance structure analysis: Conventional criteria versus new alternatives. Structural equation modeling: A multidisciplinar Journal, doi: 10.1080/10705519909540118

Huebner, E. S. (1991). Initial development of the Student's Life Satisfaction Scale. School Psychology International.

\section{doi:10.1177/0143034391123010}

Huebner, E. S. (1994). Preliminary development and validation of a multidimensional life satisfaction scale for children. Psychological Assessment. doi:10.1037/1040-3590.6.2.149

Huebner, E. S., Ash, C., \& Laughlin, J. E. (2001). Life experiences, locus of control, and school satisfaction in adolescence. Social Indicators Research. doi:10.1023/a:1010939912548.

Huebner, E. S., Drane, W., \& Valois, R. F. (2000). Levels and demographic correlates of adolescent life satisfaction reports. School Psychology International. doi: 10.1177/0143034300213005.

Huebner, E. S., \& Gilman, R. (2006). Students who like and dislike school.Applied Quality of Life Research. doi:10.1007/s11482-006-9001-3

Huebner, E. S., Gilman, R., Reschly, A., \& Hall, R. (2009). Positive schools. In S. J. Lopez (Ed.), Handbook of positive psychology (2nd Ed. pp. 445-455). New York: Plenum. doi:10.1093/oxfordhb/9780195187243.001.0001

Huebner, E. S., \& McCullough, G. (2000). Correlates of school satisfaction among adolescents. Journal of Educational Research, doi:10.1080/00220670009598725

Huebner, E., Valois, R., Paxton, R., \& Drane, J. (2005). Middle school students' perceptions of quality of life. Journal of Happiness Studies. doi:10.1007/s10902-004-1170-x

Kline, R. (2011). Principles and practice of structural equation modeling $\left(3^{\text {rd }}\right.$ ed.). New York, NY: The Guilford Press.

Lee, B. \& Yoo, M. (2015). Family, school, and community correlates of children's subjective well-being: An international comparative study. Child Indicators Research. doi:10.1007/s12187-014-9285-z

Leung, J. \& Zhang, L. (2000). Modeling life satisfaction of Chinese adolescents in Hong Kong. International Journal of Behavioral Development, 24 (1), 99-104. Recuperado de: http://journals.sagepub.com/doi/pdf/10.1080/0 16502500383520

Liu, W., Mei, J., Tian, L. \& Huebner, E. S. (2015). Age and gender differences in the relation between school-related social support and subjective well-being in school among students. Social Indicators Research. doi:10.1007/s11205-015-0873-1

Martínez-Ferrer, B., Povedano-Díaz, A., AmadorMuñoz, L. V., \& Moreno-Ruiz, D. (2012). Clima escolar, satisfacción con la vida y victimización en la escuela. Un análisis del efecto moderador del género. Anales de Psicología, 28(3), 875-882. Recuperado de: http://www.redalyc.org/articulo.oa?id=167237 74026 
McGraw, K., Moore, S., Fuller, A., \& Bates, G. (2008). Family, peer and school connectedness in final year secondary school students. Australian Psychologist. doi:10.1080/00050060701668637.

Ministerio de Educación. (2004). La Educación Chilena en el cambio de siglo: Políticas, resultados y desafios. Oficina Internacional de Educación, UNESCO. Recuperado de: www.oei.es/historico/quipu/chile/ibechile_par t1.pdf

Muthén, L., \& Muthén, B. (2013). Mplus 7.11. Los Angeles, CA: Muthén \& Muthén.

Nickerson, A. B., \& Nagle, R. J. (2004). The influence of parent and peer attachments on life satisfaction in middle childhood and early adolescence. Social Indicators Research, doi:10.1023/B:SOCI.0000007496.42095.2c.

Noddings, N. (2003).Happiness and education. Cambridge, England: Cambridge University Press.

O'Hare, W., \& Gutierrez, F. (2012). The use of domains in constructing a comprehensive composite index of child well-Being. Child Indicators Research. doi:10.1007/s12187-012-9138-6

Okun, M. A., Braver, M. W., \& Weir, R. M. (1990). Grade level differences in school satisfaction. Social Indicators Research, doi:10.1007/BF00303835

Park, N. (2004). The role of subjective well- being in positive youth development. The ANNALS of the American Academy of Political and Social Science. doi: 10.1177/0002716203260078

Park, N. (2005). Life satisfaction among Korean children and youth: A developmental perspective. School Psychology International Journal, 26, 209-223. doi: 10.1177/0143034305052914

Proctor, C., Linley, P., \& Maltby, J. (2009). Youth Life Satisfaction: A Review of the Literature. Journal of Happiness Studies. doi: 10.1007/s10902-008-9110-9.

Siyez, D., \& Kaya, A. (2008). Validity and Reliability of the Brief Multidimensional Students' Life Satisfaction Scale with Turkish Children.Journal of Psychoeducational Assessment. doi: 10.1177/0734282907307802

Smith- Castro, V., \& Molina, M. (2011).La entrevista cognitiva: Guía para su aplicación en la evaluación y mejoramiento de instrumentos de papel y lápiz. Cuadernos Metodológicos. Costa Rica: Instituto de Investigaciones Psicológicas. Recuperado de: http://filosofia.ucr.ac.cr/wpcontent/uploads/20 15/05/Cognitive-interview-2011.pdf
Steiger, J. H. (2007). Understanding the limitations of global fit assessment in structural equation modeling. Personality and Individual Differences. doi:10.1016/j.paid.2006.09.017

Suldo, S. M., \& Huebner, E. S. (2004). Does life satisfaction moderate the effects of stressful life events on psychopathological behavior during adolescence? School Psychology Quarterly. doi:10.1521/scpq.19.2.93.33313

Tian, L. (2008). Developing scale for school wellbeing in adolescents. Psychological Development and Education, 24(3), 100-106, doi:10.1007/s11205-014-0603-0

Tian, L, Chen, H., \& Huebner, E.S. (2013). The longitudinal relationships between basic psychological needs satisfaction at school and school-related subjective well-being in adolescents. Social Indicators Research, doi:10.1007/s11205-013-0495-4

Tian, L., Liu, B., Huang, S., \& Huebner, E. S. (2013). Perceived social support and school well-being among Chinese early and middle adolescents: The mediational role of selfesteem. Social Indicators Research. doi:10.1007/s1120501201238.

Tian, L., Wang, D., \& Huebner, E. (2015). Development and validation of the Brief Adolescents' Subjective Well-Being in School Scale (BASWBSS).Social Indicators Research. doi:10.1007/s11205-014-0603-0

Tian, L., Zhang, L., Huenbner, E. S., Zheng, X., \& Liu, W. (2015). The longitudinal relationship between school belonging and subjective well-being in school among elementary school students. Applied Research in Quality of Life. doi:10.1007/s11482-015-9436-5

Valois, R. F., Zullig, K. J., Huebner, E. S., \& Drane, J. W. (2004). Physical activity behaviors and perceived life satisfaction among public high school adolescents. Journal of School Health. doi:10.1111/j.1746-1561.2004.tb04201.x

Valois, R. F., Zullig, K. J., Huebner, E. S., \& Drane, J. W. (2009). Youth developmental assets and perceived life satisfaction: Is there a relationship? Applied Research Quality of Life. doi:10.1007/s11482-009-9083-9

Verkuyten, M., \& Thijs, J. (2002). Racist victimization among children in the Netherlands: The effect of ethnic group and school. Ethnic and Racial Studies. doi:10.1080/01419870120109502

Weber, M., Ruch, W., \& Huebner, E. S. (2013). Adaptation and initial validation of the german version of the Students' Life Satisfaction Scale (german SLSS). European Journal of Psychological Assessment. 
doi:10.1027/1015-5759/a000133

Whitley, A., Huebner, E. S., Hills, K., \& Valois, R. (2012). Can students be too happy in school? The optimal level of school satisfaction. Applied Research in Quality of Life. doi 10.1007/s11482-012-9167-9

Zullig, K. J., Valois, R. F., Huebner, E.S., Oeltmann, J. E., \& Drane, J. W. (2001). Relationship between perceived life satisfaction and adolescents' substance abuse. Journal of Adolescent Health, 29(4), 279-288. doi:http://dx.doi.org/10.1016/S1054139X(01)00269-5 